\title{
Comprehensive palliative treatment on Ewing sarcoma patient post forequarter amputation
}

\begin{abstract}
Introduction: Ewing's sarcoma is one of the most progressive cancer which prevalence on children and adolescent. This condition causes metastasis on other part of organ such as brain and lung. Because of it progressivity, clinical presentation can be painful, thus, decreasing quality of life and metastasis. In order to decrease the psychological burden and adverse effect, a comprehensive palliative care is needed.
\end{abstract}

Methods: We reported a a male patient aged 15 years old. The patient was diagnosed with fracture on humerus sinistra and treated by giving cast after a futsal accident .One month afterwards, swellings and pain was increased in intensity so the suspicion changed into compartment syndrome. Therefore, fasciotomy was done. A number of examinations and clinocipathological conference $(\mathrm{CPC})$ produce a conclusion Ewing's Sarcoma. The patient had finally undergone for equarter amputation surgery. Then the patient was treated with chemotherapy, however could not continue second procedure of chemotherapy as the patient died before the procedure.

Discussion: Palliative care on Ewing's sarcoma patient gives advantage when curative treatment does not work out. The progression of Ewing's sarcoma caused metastasis in other organ, the objective of palliative care itself is to prevent symptoms and minimalize the impact of treatment on quality of life. On this patient's case, the patient could be given necessary care if misdiagnosis did not happen earlier. The proper diagnosis affects outcome of the disease thus the patient got appropriate treatment and care needed.

Conclusion: Palliative therapy on patient with Ewing's sarcoma gives advantage to patient especially in terms of quality of life and life expectancy. Patient who undergo forequarter amputation will have hard time to adjust their life outside the hospital settings. Palliative therapy of choice are included surgery, radiotherapy, chemotherapy, and administration of pain-killer administration with addition of psychotherapy and medical rehabilitation.

\author{
Volume 9 Issue 5 - 2019
}

\author{
Sigit Daru Cahayadi,' Muhammad Reza \\ Saputra ${ }^{2}$ \\ 'Department of Orthopaedics and Traumatology, Persahabatan \\ Hospital, Indonesia \\ ${ }^{2}$ Orthopaedics and Traumatology Resident, Universitas \\ Indonesia-Cipto Mangunkusumo Hospital, Indonesia
}

\begin{abstract}
Correspondence: Muhammad Reza Saputra, Department of Orthopaedics and Traumatology, Persahabatan Hospital, Indonesia, Email mhrs.rezasaputra@gmail.com
\end{abstract}

Received: September 20, 2019 | Published: September 25, 2019

\section{Introduction}

Ewing sarcoma is one of the most common malignant bone tumor, primarily in children and adolescent and approximately account for $6 \%$ of childhood malignancy. ${ }^{1}$ Ewing sarcoma itself is included in Ewing's sarcoma family tumor (ESFT) included Ewing's sarcoma, peripheral primitive neuroectodermal (PNET), and Askin tumors. 2 These tumors have similarities such as developing on bone and/or soft tissues and peak incidence on young child and adolescent. ${ }^{2,3}$ Based on study from Duchman et al, we found that this tumor has most occurrence on males $(63.5 \%)$ and on below 20 years of age $(67.2 \%){ }^{4}$

Mechanism of Ewing's sarcoma is yet to be understood, however, some theories suggest that Ewing's sarcoma is derived from EWS/ETS oncogenic fusion $1 .{ }^{5}$ This will altered expression of several genes and non-coding RNA. 2,5 Tumor itself characterized with rearrangement of EWS gene on 21q22, causing translocation that occurs on $t(11 ; 22)$ (q24;q12) in progenitors or stem cells which characterized by small round blue cell tumor. ${ }^{5,6}$

Early symptoms of Ewing's sarcoma included localized pain on extremities. ${ }^{2}$ This symptoms is mostly mistaken for bone growth and injury, causing misdiagnosis and delayed in treatment. ${ }^{1,3,5}$
Progression of sarcoma increase rapidly and resulting in swelling that hard to be detected in femur, pelvis, spinal, and in chest cavity. Due to the progressivity of the disease, metastasis in organ such as brain, lung, and distant metastasis on later stage. ${ }^{7}$ The treatment for Ewing's sarcoma are included forequarter amputation for the sarcoma on upper extremities including removal of limb and scapula, chemotherapy, radiation, and molecular therapeutic target. ${ }^{5,8,9}$ Even though the treatment of Ewing sarcoma has been developing, there are side effects in quality of life such as depression, post traumatic syndrome disorder (PTSD), and other mental issues and affect the life expectancy of the patient. ${ }^{7}$ This study conducted in order to see the effect of comprehensive palliative care on Ewing's sarcoma patients who underwent forequarter amputation surgery.

\section{Case presentation}

This study is case report study including a male patient aged 15 years old diagnosed with Ewing's sarcoma. On August 2018, palpable swelling was visible on upper left arm for three months, then pain on the upper left arm appeared without any history of trauma. On September 2018, the patient was diagnosed with fracture on humerus sinistra and treated by giving cast after an accident while he was playing futsal. 
One month afterwards, swellings and pain was increased in intensity so the suspicion changed into compartment syndrome. Therefore, fasciotomy was done and histopathological examination was used to diagnose with suspicion of small round cell. Patient then was referred to Rumah Sakit Persahabatan for work up diagnostic. After MRI examination, histopathological result, and clinico pathological conference (CPC), patient was diagnosed with Ewing's sarcoma on upper left arm. On November 2018, patient were finally undergone forequarter amputation surgery. Decrease of weight also being reported. When physical examination conducted for the first time, all signs were normal, except the appearance of mass and wound without active bleeding on posterolateral side of upper left arm with limited ROM on left elbow, wrist, and thumb. Mass was palpable with diameter of $32 \mathrm{~cm}$ (contralateral diameter $17 \mathrm{~cm}$ ), hypoesthesia on radial, and VAS level 4-5. Histopathological anatomy shown appearance of small blue tumor cells. The clinical presentation, laboratory result, and MRI result were shown on Table 1 and Figure $1-3$.

Table I Laboratory result of patient with Ewing's sarcoma

\begin{tabular}{llll}
\hline Haemoglobin & 10.2 & $\mathrm{~g} / \mathrm{dL}$ & $13.0-16.0$ \\
Haematocrit & 30.7 & $\%$ & $40.0-48.0$ \\
Erythrocyte & 3.59 & $10^{\wedge} 6 / \mu \mathrm{L}$ & $4.50-5.50$ \\
Thrombocyte count & 729 & $10^{\wedge} 3 / \mu \mathrm{L}$ & $150-400$ \\
Leucocyte count & 16.22 & $10^{\wedge} 3 / \mu \mathrm{L}$ & $5.00-10.00$ \\
RDW-CV & 13.2 & & $11.5-14.5$ \\
Ureum & 26 & $\mathrm{mg} / \mathrm{dL}$ & $18-55$ \\
SGPT (ALT) & 84 & $\mathrm{U} / \mathrm{L}$ & $5-34$ \\
SGOT (AST) & 81 & $\mathrm{U} / \mathrm{L}$ & $0-55$ \\
Phosphatase alkaline & 149 & $\mathrm{U} / \mathrm{L}$ & $40-150$ \\
LDH & 2601 & $\mathrm{mEq} / \mathrm{L}$ & $98.0-107.0$ \\
Blood creatinine & 0.7 & $\mathrm{mg} / \mathrm{dL}$ & $0.6-1.2$ \\
\hline
\end{tabular}

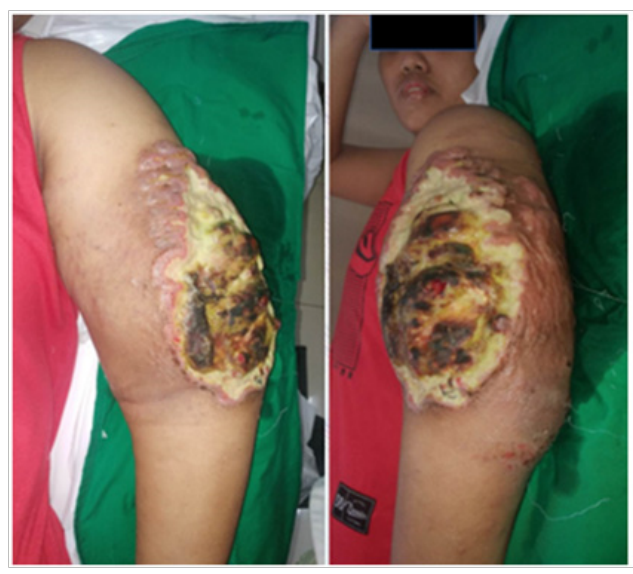

Figure I Clinical presentation of patient.

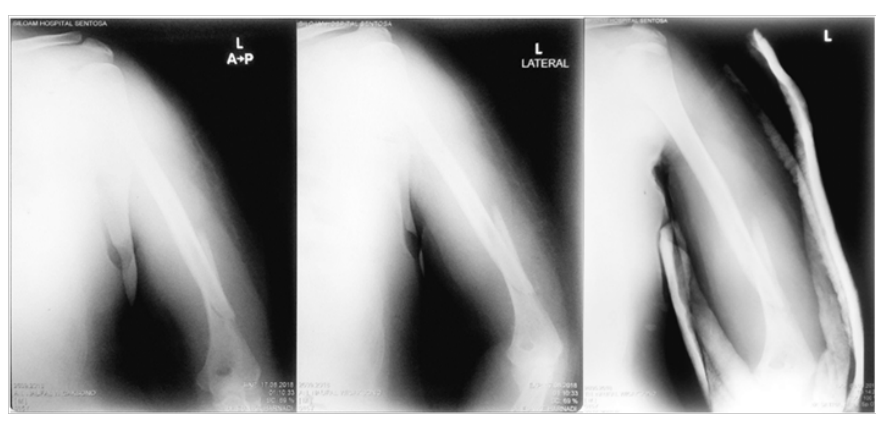

Figure 2 Rontgen of left humerus.

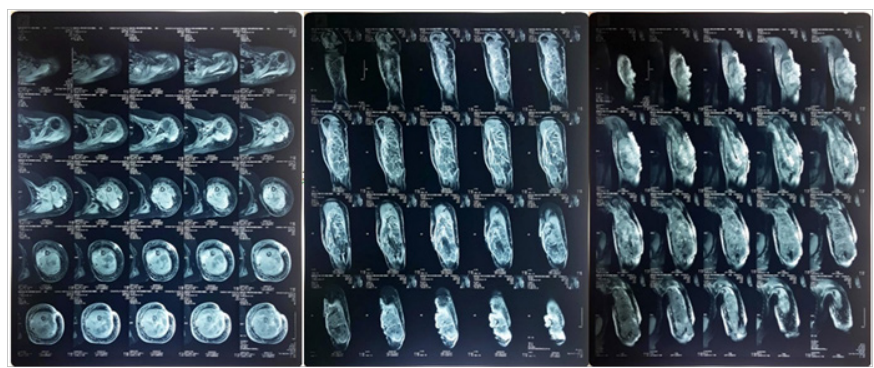

Figure $3 \mathrm{MRI}$ of humerus sinistra.

From the test, we found that instead of fracture on humerus, the diagnosis of the patient is closed-pathological fracture of left humerus due to primary bone tumor of left shaft humerus suspect malignant with differential diagnosis Ewing Sarcoma. After the diagnosis and amputation, patient was treated with chemotherapy due to pleural effusion and metastasis, however the patient could not continue second procedure of chemotherapy as the patient died before the procedure.

\section{Discussion}

Palliative care on Ewing's sarcoma patient gives advantage when curative treatment does not work out. Without proper care, patient may experience psychological adverse effect. Study from Wiener et al used 34 pediatric sarcoma survivor who are completing SCID module for PTSD. The study shows that $12 \%$ patient met criteria of PTSD and associated with intrusive thought, avoidant behavior, and difficulties on adjusting normal life such as home, school, or work. ${ }^{10}$

While the progression of Ewing's sarcoma caused metastasis in other organ, the objective of palliative care itself is to prevent symptoms and minimalize the impact of treatment on quality of life. ${ }^{11}$ Study from Koontz et al. ${ }^{11}$ to 21 patients with metastatic Ewing's sarcoma received palliative radiotherapy shows that one patients who shows metastatic on brain has been disease free for 3.4 years after resection while the other patient died due to their disease. The survivor lived for a median duration of one year after metastatic diagnosis. All of the patient has median response duration 4 months, ranging from 10 days to 4.8 years. ${ }^{11}$ Another study conducted by Wittig et al which used 8 patients who underwent palliative forequarter amputation. All patients were given epineural catheters of bupivacaine infusion for pain control. From the study, we found that all patients experienced relieve and improvement of body function without phantom limb pain and psychological reaction. ${ }^{9}$ Another study from Goefert et al. ${ }^{12}$ was using 19 patients with Ewing's sarcoma who were given therapy 
such as radiotherapy and chemotherapy. From 19 patients, 2 were considered free from the disease respectively twelve months and forty eight months after therapy. Two patients then were given adjuvant therapy. ${ }^{12}$

Study from Sofyer et al used 17 patient with symptomatic metastatic sarcoma who are on median age of 61 years who underwent CT simulation and given intensive regiment of $39 \mathrm{~Gy}$ in 13 fractions of $3 \mathrm{~Gy} /$ day, 5 times a week. The patient then were followed up after 25 weeks. At the end of the follow up period, 14 patients were survived. ${ }^{13}$

In order to regain function in patient with debilitating impairment caused by amputation or cancer malignancy and decrease the burden of care required by patient, medical rehabilitation is also needed. During acute post-surgical phase, return-to-school and return to work strategy should be considered alongside with referral to appropriate rehabilitation center and assistive or orthotic equipment. By 6 weeks or beyond as needed, physical therapy such as increasing the girth of affecting limb, discontinuing using assistive tools, and improving endurance on the limb affected. ${ }^{14}$ An observational study conducted by Strukelj et al. ${ }^{15}$ used 60 oncological amputees while rehabilitation outcome was tested with Functional Independence Measurement (motor FIM, max 91). From the study, we can find that 50\% low limb amputee has life expectancy for over 5 years and younger patients were able to walk faster and make longer distance. ${ }^{15}$

Psychological care plays a vital role since a patient should understand the disease status in order to prepare them mentally and emotionally to face their situation and outcome of their treatment and disease. ${ }^{7}$ As for a patient with amputation reintegrates and readjusts their live, emotional burden becomes such a challenge for patient, thus psychotherapy is needed in order to decrease emotional burden of sarcoma patient, especially post amputation patient. A study conducted by Srivastava et al by using 173 patients with limb amputations. All the patient were tested by questionnaires such as Carroll Rating Scale for Depression (CRSD), State-Trait Anxiety Inventory (STAI), Amputees Body Image Scale (ABIS), and Impact of Event Scale (IES) along with specially designed information schedule. A structured psychotherapy session was also designed for patient. After two months of therapy session, improvement on the Carroll Rating Scale of Depression, in the PIM group, initial scores were 16.08. Therapeutic intervention reduced the score to 10.19. On the other hand, in the TAU group there was reduction of CRSD scores from 15.87 to 14.39 (differences not statistically significant). Similar result also shown in anxiety assessment on PIM group (42.26 to 35.12) with $\mathrm{p}<0.05$ while in TAU group, improvement existed, however it is not statistically significant. (42.38 (7.09) to 40.16 (6.94), $\mathrm{p}>0.05){ }^{16}$

A study by Sachsenmaler et al. ${ }^{17}$ on 169 patients with soft tissue or bone sarcoma who were given questionnaire to measure their quality of life following surgery of the cancer. From the study, 53\% patient showed optimism in the future and has better physical status. It also shown that consultations with the psycho oncological service during treatment was positively associated with current happiness $(\mathrm{n}=56, \mathrm{P}=0.0029)$. This concludes that stable emotional situation is significantly related with better prognosis of the patients and physical outcomes with the help of psychological care. ${ }^{17}$

On this patient's case, the patient could be given necessary care if misdiagnosis did not happen earlier. The proper diagnosis affects outcome of the disease thus the patient got appropriate treatment and care needed. However, it is difficult to diagnose malignancy since the clinical presentation may similar to other disease.

\section{Conclusion}

From all the study above, it can be concluded that palliative therapy on patient with Ewing's sarcoma gives advantage to patient especially in terms of quality of life and life expectancy. Patient who undergo forequarter amputation will have hard time to adjust their life outside the hospital settings. Palliative therapy of choice are included radiotherapy, chemotherapy, and administration of painkiller administration with addition of psychotherapy and medical rehabilitation.

\section{Acknowledgments}

None.

\section{Funding}

None.

\section{Conflict of interest}

There is no conflict of interest.

\section{References}

1. Heare T, Hensley M, Dell'Orfano S. Bone tumors: osteosarcoma and Ewing's sarcoma. Curr Opin Pediatr. 2009;21(3):365-372.

2. Riggi N, Stamenkovic I. The Biology of Ewing sarcoma. Cancer Lett 2007;254(1):1-10.

3. Gaspar N, Hawkins DS, Dirksen U, et al. Ewing sarcoma: Current management and future approaches through collaboration. Journal of Clinical Oncology. 2015;33(27):3036-3046.

4. Duchman KR, Gao Y, Miller BJ. Prognostic factors for survival in patients with high-grade osteosarcoma using the Surveillance, Epidemiology, and End Results (SEER) Program database. Cancer Epidemiol. 2015;39(4):593-599.

5. Rani PSV, Shyamala K, Girish HC, et al. Pathogenesis of Ewing sarcoma: A review. J Adv Clin Res Insights. 2016;2:164-168.

6. Smith SR. Rehabilitation Strategies and Outcomes of the Sarcoma Patient. Phys Med Rehabil Clin N Am. 2017;28(1):171-80.

7. Gulia A, Byregowda S, Panda P. Palliative care in musculoskeletal oncology. Indian J Palliat Care. 2016;22(3):244-251.

8. Karakousis CP, Butsch JL. Forequarter amputation. Ann Surg Oncol. $2011 ; 18(3): 726$.

9. Wittig JC, Bickels J, Kollender Y, et al. Palliative forequarter amputation for metastatic carcinoma to the shoulder girdle region: Indications, preoperative evaluation, surgical technique, and results. J Surg Oncol. 2001;77(2):105-113.

10. Wiener L. Long-Term Survivors of Pediatric Sarcoma : the Experience At a Single Institution. Psychooncology. 2006;15(10):898-910.

11. Koontz BF, Clough RW, Halperin EC. Palliative radiation therapy for metastatic Ewing sarcoma. Cancer. 2006;106(8):1790-1793.

12. Goepfert H, Rochlin DB, Smart CR, et al. Palliative Treatment of Ewing' s Sarcoma. Am J Surg. 1967;113(2)::246-250.

13. Corn BW, Merimsky O, Soyfer V, et al. Radiation Therapy for Palliation of Sarcoma Metastases: A Unique and Uniform Hypofractionation Experience. Sarcoma. 2010;2010:1-4.

14. Jaffe N. Pediatric and Adolescent Osteosarcoma. 2009;1-591.

15. Metka Prešern S, U Zupanc, M Zalar. Rehabilitation of Oncological Amputee Patients. J Heal Sci. 2015;3(2):81-84. 
16. Srivastava K, Chaudhury S. Rehabilitation after Amputation: Psychotherapeutic Intervention Module in Indian Scenario. Sci World J. 2014.
17. Sachsenmaier SM, Ipach I, Kluba T. Quality of life, physical and mental status and contentment of patients with localized soft tissue or bone sarcoma: A questionnaire analysis. Orthop Rev (Pavia). 2015;7(2):5920. 